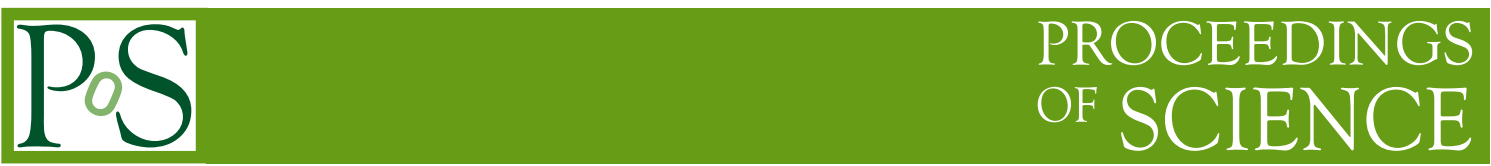

\title{
New AGNs discovered by H.E.S.S.
}

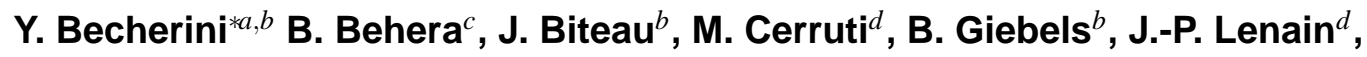 \\ M. de Naurois ${ }^{b}$, M. Punch ${ }^{a}$, M. Raue ${ }^{e}$, D. Sanchez ${ }^{f}$, F. Volpe ${ }^{f}, \mathbf{A}$. Zech $^{d}$ on behalf of \\ the H.E.S.S. Collaboration \\ ${ }^{a}$ Astroparticule et Cosmologie (APC), CNRS, Université Paris Diderot - Paris 7, Paris, France \\ ${ }^{b}$ Laboratoire Leprince-Ringuet, Ecole Polytechnique, CNRS/IN2P3, Palaiseau, France \\ ${ }^{c}$ Landessternwarte, Universitat Heidelberg, Heidelberg, Germany \\ ${ }^{d}$ Laboratoire Univers et Theories (LUTH), Observatoire de Paris, CNRS, Université Paris \\ Diderot-Paris 7, Meudon, France \\ ${ }^{e}$ Universitat Hambourg, Institut fur Experimentalphysik, Hambourg, Germany \\ ${ }^{f}$ Max-Planck-Institut fur Kernphysik, Heidelberg, Germany \\ E-mail: Yvonne.Becherini@apc.univ-paris7.fr
}

During the last year, six new Active Galactic Nuclei (AGN) have been discovered and studied by H.E.S.S. at Very High Energies (VHE). Some of these recent discoveries have been made thanks to new enhanced analysis methods and are presented at this conference for the first time. The three blazars 1ES 0414 + 009, SHBL J001355.9 - 185406 and 1RXS J101015.9 - 311909 have been targeted for observation due to their high levels of radio and X-ray fluxes, while the Fermi/LAT catalogue of bright sources triggered the observation of PKS 0447 - 439 and AP Librae. Additionally, the BL Lac 1ES 1312 - 423 was discovered in the field-of-view (FoV) of Centaurus A thanks to the large exposure dedicated by H.E.S.S. to this particularly interesting source. The newly-discovered sources are presented here and in three companion presentations at this conference.

25th Texas Symposium on Relativistic Astrophysics - TEXAS 2010

December 06-10, 2010

Heidelberg, Germany

\footnotetext{
* Speaker.
} 


\section{Introduction}

Eight years of operation of the High Energy Stereoscopic System (H.E.S.S.) have been at the heart of a breakthrough in the field of $\gamma$-ray VHE astronomy: more than $70 \gamma$-ray sources have been discovered, 30 of which are extra-galactic. H.E.S.S. will continue operation while awaiting the completion of the ongoing upgrade of the array, which will lead to the phase II of the experiment (see Sec. 8 for more details). Meanwhile, H.E.S.S. is improving its sensitivity also through the development and successful application of new enhanced analysis methods, such as for instance [1] and [2]. These new analysis methods allow the detection of new faint $\gamma$-ray sources, having very low flux levels, i.e. of the order of $1 \%$ C.U. ${ }^{1}$ or less, in a moderate amount of observation time. Six newly-discovered AGNs will be presented here, the detections of two of which (1RXS J101015.9-311909 and 1ES 1312-423) are announced at this conference for the first time.

H.E.S.S. observation campaigns on AGNs have been largely based in the past on the criterion proposed in [3], where, briefly, a source is considered a good VHE candidate if it exhibits already high levels of both X-ray and radio emissions. More recently, a new complementary observation strategy is in place since the advent of the Fermi/LAT telescope, in which AGNs are targeted if the extrapolation of the $\mathrm{GeV}$ spectra (e.g. as given in [4]) to higher energies - taking into account the $E B L^{2}$ absorption as a function of redshift - indicate that they may have a detectable counterpart in the VHE domain. The spectra of distant blazars are modified by absorption of the VHE $\gamma$-rays on the EBL, with an effect which depends on the distance to the source and the EBL level assumed. Correspondingly, for a source at a known distance, if the instrinsic spectrum is assumed (e.g. by extrapolation from lower energies), then a limit can be placed on the EBL level, by evaluating the absorption required to produce the measured spectrum.

The two observation strategies above led to the recent discovery of several new AGNs of the BL Lacertae ${ }^{3}$ (BL Lac or blazar) type. The three blazars 1ES 0414 + 009, SHBL J001355.9 185406 and 1RXS J101015.9 - 311909 were targeted for observations following [3], while the Fermi/LAT catalogue criterion motivated the observation of PKS 0447 - 439 and AP Lib. Moreover, low-redshift X-ray blazars were also proposed to be good candidates for VHE astronomy by [5], and hence some such were also included in the list of possible interesting targets. 1ES $1312-$ 423 is one such AGN, whose location is about $2^{\circ}$ from the position of the radio-galaxy Centaurus A, a source which has been extensively observed by H.E.S.S. and discovered in VHE $\gamma$-rays.

Table 1 summarizes the results presented at this conference in this and the companion proceedings (see references in the table). The main characteristics of the AGNs presented (position, redshift, flux level, mean observing angle, number of standard deviations of the detection, observing strategy) are given, together with the corresponding good quality livetime, which represents

\footnotetext{
1"Crab Units", i.e., compared to a hypothetical source with the Crab nebula intensity and spectrum at the zenith angle of observation.

${ }^{2}$ Extra-Galactic Background Light, resulting from the combined emission of stars and galaxies from the earliest times of their formation to the present.

${ }^{3} \mathrm{BL}$ Lac objects are generally characterized by relativistic jets beamed towards the observer, which are thought to originate the large and rapid variability seen in all energy ranges. Additionally, these objects show high polarization and lack of emission lines.
} 


\begin{tabular}{|l|c|c|c|c|c|c|c|c|}
\hline \hline Source name & RA & Dec & $z$ & LT & $\phi_{0}$ & $\theta_{\text {zen }}$ & $\mathrm{N}_{\sigma}$ & Strat. \\
\hline \hline 1ES 0414+009 [8] & 64.22 & 1.08 & 0.287 & 74 & $0.5 \%$ & $26^{\circ}$ & 7.8 & {$[3]$} \\
SHBL J001355.9-185406 & 3.48 & -18.90 & 0.095 & 38 & $\sim 1 \%$ & $13^{\circ}$ & $\sim 5$ & {$[3]$} \\
PKS 0447-439 [18] & 72.35 & -43.84 & {$[0.176,0.5]$} & 14 & $4.5 \%$ & $23^{\circ}$ & 14 & {$[4]$} \\
AP Lib [20] & 229.42 & -24.37 & 0.049 & 11 & $2 \%$ & $13^{\circ}$ & 7 & {$[4]$} \\
1RXS J101015.9-311909 & 152.57 & -31.32 & 0.14 & 33 & $\sim 2.5 \%$ & $13^{\circ}$ & 7.2 & {$[3]$} \\
1ES 1312-423 & 198.76 & -42.61 & 0.105 & $168^{*}$ & $0.4 \%$ & $24^{\circ}$ & 6.8 & {$[5]$} \\
\hline \hline
\end{tabular}

Table 1: The six new AGNs detected by H.E.S.S. during the past year. The columns present the source name and corresponding references, position in right ascension and declination in degrees, the redshift $z$, observation livetime in hours, flux in C.U., mean observing angle in degrees, significance in number of standard deviations, and finally the references used to guide the observing strategies. The sources detailed in other proceedings at this conference, have this reference in bold besides their names. (*) For 1ES 1312 - 423 this is the total livetime, but correcting for the camera acceptance this reduces to about 65 hours.

the amount of good quality data after weather selection criteria and corrected for the instrument dead-time.

\section{1ES 0414 + 009: one of the most distant VHE blazars with a known redshift}

1ES $0414+009$ is one of the distant sources having a known redshift of about $\sim 0.29$, which has been detected by H.E.S.S. with a significance of about $7.8 \sigma$ after a four-year observing campaign, leading to a total good livetime of about 74 hours (see [8] at this conference). The detection of this faint source $(0.5 \%$ C.U.) occurred at the same time the Fermi/LAT found its GeV emission by analyzing the 21 months dataset; this led to a Astronomer's Telegram [7] in which the discovery of the GeV-TeV emissions was announced simultaneously. This AGN is a high-frequency-peaked BL Lac (HBL), where the high-energy part of the spectral energy distribution (SED) shows a peak of the emission above a few TeV. Such a feature is difficult to explain with a standard one-zone SSC scenario, therefore, this source belongs to the class of the hard TeV BL Lac objects, which are challenging for modelling.

\section{Discovery of SHBL J001355.9 - 185406: first H.E.S.S., then Fermi/LAT}

The newly-discovered SHBL J001355.9 - 185406, also belonging to the HBL category, is much closer, with a redshift of 0.095 . The source is contained among the sources of the RASS/BSC ${ }^{4}$ of soft $(0.1-2 \mathrm{keV}) \mathrm{X}$-ray sources (see [9]), where its flux was found to be $1.01 \times 10^{-11} \mathrm{erg} / \mathrm{cm}^{2} / \mathrm{s}$ and is in the NVSS catalogue ${ }^{5}$ of radio sources at $1.4 \mathrm{GHz}$ (see [10]) which gives its flux density at $29.5 \mathrm{mJy}$. The source was selected according to the criterion in [3], but also from the list of 150 HBL sources in the $\mathrm{SHBL}^{6}$ catalogue (see [11]), where, briefly, interesting VHE targets show an extremely high ratio of X-ray to radio fluxes. The source was not present in the first year

\footnotetext{
${ }^{4}$ ROSAT All Sky Survey Bright Source catalogue (18811 sources)

${ }^{5}$ NRAO (National Radio Astronomy Observatory) VLA (Very Large Array) Sky Survey $\left(1.8 \times 10^{6}\right.$ sources)

${ }^{6}$ Sedentary High-frequency BL Lacertae
} 
Fermi/LAT catalogue, indicating that its flux in the GeV range was below the 1-year sensitivity. H.E.S.S. discovered the VHE emission from this BL Lac object [12] using the two advanced analysis methods mentioned in Sec. 1, with a statistical significance of about $5 \sigma$ above $300 \mathrm{GeV}$ in 38 hours of good quality observations carried out between July 2008 and August 2010, see Fig. 1a for the excess map. The light curve of the source is found to be compatible with a constant flux at the level of $\sim 1 \%$ C.U. The source was monitored in optical wavelengths over the same period by $\mathrm{ATOM}^{7}$, operated by the H.E.S.S. collaboration and located next to the H.E.S.S. site. Additionally, Swift/XRT [13] observations in the $0.2-10 \mathrm{keV}$ energy range were triggered by H.E.S.S. in September 2010. No significant variability in either optical or X-ray bands down to a timescale of a few days has been found. Subsequent to the H.E.S.S. discovery, the Fermi/LAT Collaboration announced the detection of a source positionally consistent with the BL Lac with a significance of $7 \sigma$, see [14]. The Fermi/LAT preliminary analysis indicated a faint hard-spectrum source with a 2year averaged flux above $100 \mathrm{MeV}$ of $(0.9 \pm 0.7) \times 10^{9} \mathrm{ph} / \mathrm{cm}^{2} / \mathrm{s}$ and a photon index of $1.5 \pm 0.2$, considering statistical errors only.

\section{PKS $0447-439$ : trying to constrain the redshift}

PKS $0447-439$ is one of the brightest hard-spectrum blazars in the Fermi/LAT catalogue. H.E.S.S. observed this source for a total livetime of about 14 hours, announcing the discovery of the VHE $\gamma$-ray emission in [15] with a significance of $14 \sigma$. The redshift of this source is not well known; a value of 0.205 was initially proposed by [16], whereas a more recent study [17] provides only a lower limit of 0.176 . The study detailed in [18] at this conference reverses the procedure for the evaluation of the EBL level discussed in Sec. 1. It supposes the EBL to be now known with some certitude and explores the range of acceptable redshifts for which the extrapolation of the spectrum from the Fermi/LAT range does not overproduce the predicted spectrum in the H.E.S.S. range, within measurement errors. In this way, an upper limit in redshift of 0.5 was derived at the $95 \%$ confidence level.

\section{AP Librae: first LBL of the southern hemisphere}

AP Librae (AP Lib) is the closest of the new blazars presented here, at a redshift of 0.049 , and was targeted for H.E.S.S. observations thanks to the Fermi/LAT bright source catalogue. This AGN has been discovered by H.E.S.S. after 11 hours of livetime with a significance of $7 \sigma$ and announced in [19]. The SED (see [20], at this conference) of this source shows that AP Lib is a low-frequency peaked BL Lac (LBL) and presents an extremely broad high-energy component which extends from X-ray to VHE, showing that, for this source, synchrotron X-ray brightness is not required to produce VHE $\gamma$-rays and that an external Compton component may be responsible for detected VHE emission. AP Lib is the third LBL detected at VHE and the first one discovered in the southern hemisphere.

\footnotetext{
${ }^{7}$ Automatic Telescope for Optical Monitoring
} 

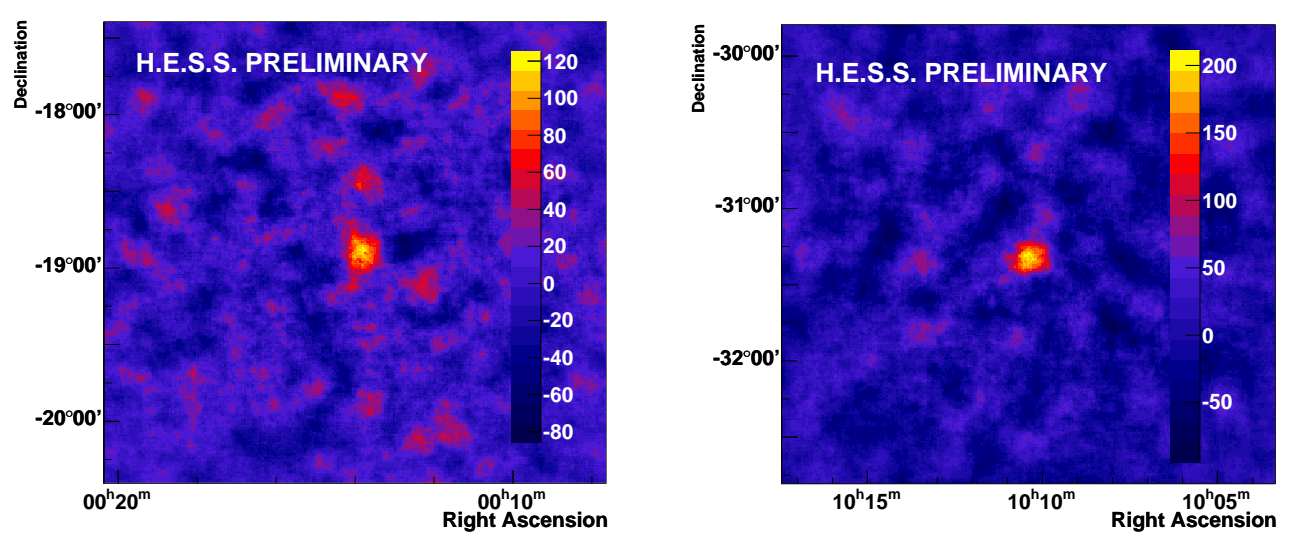

Figure 1: Left panel. Excess map of the region including the blazar SHBL J001355.9 - 185406. Right panel. Excess map of the region including the blazar 1RXS J101015.9-311909.

\section{Discovery of VHE $\gamma$-rays from the BL Lac object 1RXS J101015.9-311909}

1RXS J101015.9 - 311909 is a blazar at a redshift of 0.14. The source is a HBL contained in the RASS/BSC catalogue of soft X-ray sources, where its flux is given as $2.9 \times 10^{-11} \mathrm{erg} / \mathrm{cm}^{2} / \mathrm{s}$ and is in the NVSS catalogue of radio sources at $1.4 \mathrm{GHz}$, where its flux density is $74.3 \mathrm{mJy}$. This AGN has been shown to be a good candidate for VHE Cherenkov astronomy also for its extremely high X-ray to radio flux ratio, pointed out in the SHBL catalogue, and was not present in the first year Fermi/LAT catalogue ([4]). H.E.S.S. observed this AGN in a dedicated campaign between 2007 and 2010 at a mean observing angle of $13^{\circ}$, leading to a total good livetime of 33 hours. The analysis of this AGN was carried out with the two independent enhanced analysis procedures mentioned in Sec. 1, which give consistent results. VHE emission from the BL Lac object 1RXS J101015.9 - 311909 has thus been discovered by H.E.S.S. with an excess of 233 $\gamma$-ray events, at a significance level of $7.2 \sigma$, and is presented at this conference for the first time. The VHE $\gamma$-ray excess sky map is shown in Fig. 1b. The flux of the source is about $2.5 \%$ C.U., and spectral studies are ongoing.

\section{Discovery of 1ES 1312 - 423: in the same FoV of Centaurus A}

1ES $1312-423$ is a blazar at a redshift of 0.105 present in the Einstein slew survey sample [21], in the RASS/BSC, and in the BeppoSAX spectral survey of BL Lacs [22]. The source was proposed as a good VHE $\gamma$-ray candidate by [5], where it was suggested through a simple SSC model that low-redshift X-ray BL Lac objects should have a VHE counterpart. The source was not present in the Fermi/LAT first year catalogue. 1ES 1312-423 lies at the edge of the FoV of H.E.S.S. for observations centred around the radio-galaxy Centaurus A. H.E.S.S. carried out a deep observation of Centaurus A leading to its discovery in the VHE domain in early 2009 ([6]), reporting a flux level of $0.8 \%$ in C.U. For this reason, the data set available for the entire FoV is large, and has been useful to perform a dedicated analysis on this blazar, despite the lower collection efficiency at about $2^{\circ}$ distance from the observing position. The source has been analyzed with the 


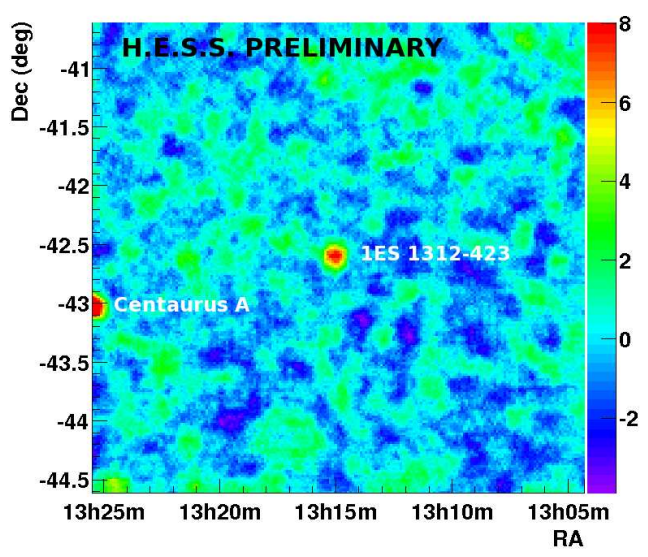

Figure 2: Significance map of $1 \mathrm{ES} 1312-423$. The source on the left-hand side is the radio-galaxy Centaurus A.

two above-mentioned enhanced analysis methods leading to the the discovery of its VHE emission with a $6.8 \sigma$ significance in 168 hours of good livetime taken between 2004 and 2010. The total corrected livetime, taking into account that the observations were carried out at large offset with respect to the source position, is of about 65 hours. Preliminary studies of the light curve show no detectable variability, similarly to observations in other wavelengths, which were not variable either.

\section{Conclusions}

H.E.S.S. is now in the process of refurbishing the four mirror surfaces and building a new very-large telescope, in preparation for the operation of its second phase, which will begin in 2012. The new telescope will permit to lower the threshold to about $30 \mathrm{GeV}$ in the single-telescope mode, opening a complementary observational window to high-energy phenomena. As negligible $\gamma$-ray absorption is expected in the $30-100 \mathrm{GeV}$ energy range, therefore more distant objects will become visible, enriching the extra-galactic source catalogue and permitting to put stronger constraints on the EBL. By enriching the array with the addition of a fifth telescope at the centre, the experiment will be about a factor of two more sensitive with respect to the present configuration in multi-telescope mode. In the meantime, H.E.S.S. has succeeded in enhancing its sensitivity uniquely by the development and successful application of new, more powerful analysis methods. Thanks to the enhanced sensitivity provided by these new methods, sources which were not seen with the standard analyses, became detectable. At this conference, six newly-detected AGNs have been presented, the discovery of two of which (1RXS J101015.9-311909 and 1ES 1312 - 423) has been shown for the first time. While the detections of PKS 0447 - 439 and AP Lib were motivated by the Fermi/LAT catalogue of bright sources, the discovery of SHBL J001355.9 - 185406 was first achieved at VHE by H.E.S.S., and subsequently by Fermi/LAT, integrating over a larger dataset. The SEDs of 1ES $0414+009$ and AP Lib have peculiar features which are difficult to explain with standard one-zone SSC scenarios, giving intriguing inputs for the modelling of LBLs and HBLs. With the detection of 1ES $0414+009$ and 1ES $1312-423$, two blazars having flux levels of $0.5 \%$ 
and $0.4 \%$ C.U., H.E.S.S. enriches the catalogue of the weakest-ever sources detected in VHE $\gamma$-rays. These recent discoveries demonstrate that the field of extragalactic VHE $\gamma$-ray astronomy is very active and will become more so with the advent of the second phase of H.E.S.S.: HESS-II.

\section{Acknowledgements}

The support of the Namibian authorities and of the University of Namibia in facilitating the construction and operation of H.E.S.S. is gratefully acknowledged, as is the support by the German Ministry for Education and Research (BMBF), the Max Planck Society, the French Ministry for Research, the CNRS-IN2P3 and the Astroparticle Interdisciplinary Programme of the CNRS, the U.K. Science and Technology Facilities Council (STFC), the IPNP of the Charles University, the Polish Ministry of Science and Higher Education, the South African Department of Science and Technology and National Research Foundation, and by the University of Namibia. We appreciate the excellent work of the technical support staff in Berlin, Durham, Hamburg, Heidelberg, Palaiseau, Paris, Saclay, and in Namibia in the construction and operation of the equipment.

\section{References}

[1] M. de Naurois \& L. Rolland, Astroparticle Physics 32, 231 - 252, 2009

[2] Y. Becherini et al., A new analysis strategy for detection of faint gamma-ray sources with Imaging Atmospheric Cherenkov Telescopes, Astropart. Phys. (2011), doi:10.1016/j.astropartphys.2011.03.005

[3] L. Costamante and G. Ghisellini, A\&A, 384, 56, 2002

[4] A.A. Abdo et al. (2010), ApJS 188, 405, 2010

[5] F.W. Stecker et al., The Astrophysical Journal, 473, 75 - 78, 1996

[6] F. Aharonian et al., Astrophys. J. Lett. 695, 40-44, 2009

[7] W. Hofmann on behalf of the H.E.S.S. Collaboration and S. Fegan on behalf of the Fermi/LAT Collaboration, Astronomer's Telelegram \#2293, 2009

[8] F. Volpe et al. on behalf of the H.E.S.S. Collaboration and L. Costamante et al. on behalf of the Fermi/LAT Collaboration, Discovery of VHE and HE emission from the blazar 1ES $0414+009$ with H.E.S.S. and Fermi/LAT, at this conference

[9] W. Voges et al., A\&A 349, 389-405, 1999

[10] J.J. Condon et al., The Astronomical Journal, 115 - 5, 1693 - 1716, 1998

[11] P. Giommi et al., A\&A, 434, 385, 2005

[12] W. Hoffmann on behalf of the H.E.S.S. Collaboration, Astronomer's Telegram 3007, 2010

[13] http://heasarc.nasa.gov/docs/swift/swiftsc.html

[14] D. Sanchez et al., for the Fermi/LAT Collab., Astronomer's Telegram 3023, 2010

[15] M. Raue et al. on behalf of the H.E.S.S. Collaboration, Astronomer's Telegram \#2350, 2009

[16] E.S. Perlman et al., ApJ, 115, 1253, 1998 
[17] H. Landt \& H.E. Bignall, MNRAS, 391, 967, 2008

[18] A. Zech et al. on behalf of the H.E.S.S. Collaboration, Discovery of VHE emission from PKS 0447 - 439 with H.E.S.S. and MWL studies, at this conference

[19] W. Hofmann on behalf of the H.E.S.S. Collaboration, Astronomer's Telegram \#2743, 17 Jul 2010

[20] P. Fortin et al., on behalf of the Fermi/LAT Collaboration and B. Giebels et al. on behalf of the H.E.S.S. Collaboration, Gamma-ray emission from AP Librae (PKS 1514-241), at this conference

[21] E. Perlman et al., ApJS, 104, 251, 1996

[22] V. Beckmann et al., A\&A 383, 410-422, 2002 